\title{
The Role of Visual and Body Movement Information in Infant Search
}

\author{
Mark A. Schmuckler and Hannah Y. Tsang-Tong \\ University of Toronto at Scarborough
}

\begin{abstract}
Three experiments investigated the use of visual input and body movement input arising from movement through the world on spatial orientation. Infants between $91 / 2$ and 18 months participated in a search task in which they searched for a toy hidden in 1 of 2 containers. Prior to beginning search, either the infants or the containers were rotated $180^{\circ}$; these rotations occurred in a lit or dark environment. These experiments were distinguished by the environmental cues for object location; Experiment 1 used a position cue, Experiment 2 a color cue, and Experiment 3 both position and color cues. Accuracy was better in Experiments 2 and 3 than in Experiment 1. All studies found that search was best after infant movement in the light; all other conditions led to equivalently worse performance. These results are discussed relative to a theoretical characterization of spatial coding focusing on the uses of spatial information.
\end{abstract}

One crucial cognitive skill to emerge over the first few years of life is the ability to orient spatially within the environment. One example of this skill involves an infant's conception of the existence of an object hidden from view and the ability to keep track of the location of this hidden object. Piaget (1954) provided the classic example of this ability in his observation that an infant will search for a hidden object at the location at which it had been previously found, despite seeing the object being hidden at a different location (the Stage IV, A not B error). This result has spurred a great deal of research focusing on the child's conception of objects (e.g., Baillargeon, 1987; Baillargeon, DeVos, \& Graber, 1989; Baillargeon \& Graber, 1988; Bower \& Paterson, 1971; Evans \& Gratch, 1972; Gratch, Appel, Evans, LeCompte, \& Wright, 1974; Gratch \& Landers, 1971) and the child's spatial knowledge (e.g., Aymed \& Ruffman, 1998; Benson \& Uzgiris, 1985; Bremner, Knowles, \& Andreasen, 1994; Butterworth, 1976, 1977; Hofstadter \& Reznick, 1996; Lucas \& Uzgiris, 1977; Smith, Thelen, Titzer, \& McLin, 1999).

Piaget (1954; Flavell, 1963) assumed that failures in finding this object indicated the limited nature of the child's conception of objects. Along these lines, objects became linked to a specific physical action occurring at a specific locale defined with refer-

Editor's Note. Richard Aslin served as the action editor for this manuscript.-JLD

Mark A. Schmuckler and Hannah Y. Tsang-Tong, Division of Life Sciences, University of Toronto at Scarborough, Scarborough, Ontario, Canada.

Hannah Y. Tsang-Tong is now in medical practice in Hong Kong.

This research was supported by Natural Sciences and Engineering Research Council of Canada Grant OGPO089706. We thank Bennett Bertenthal, Phil Zelazo, and three anonymous reviewers for their helpful comments on earlier versions of this article.

Correspondence concerning this article should be addressed to Mark A. Schmuckler, Division of Life Sciences, University of Toronto at Scarborough, 1265 Military Trail, Scarborough, Ontario, Canada M1C 1A4. Electronic mail may be sent to marksch@banks.scar.utoronto.ca. ence to one's position in space, making the conception of the object and the spatial world "egocentric." As an alternative, spatial orientation could be relative to an external frame; such coding has been termed "allocentric" (Bremner, 1978a, 1978b; Bremner \& Bryant, 1977) and requires updating one's conception of one's position in space relative to stable landmarks within the environment.

One common assumption of this conceptualization of spatial orientation is that changes in a child's spatial abilities reflect qualitative differences in the underlying representational system used by the child. Thus, the child begins life using an egocentric representation that localizes objects relative to one's own body, with little understanding of one's position in space or the environment. Such egocentric coding is thought to predominate spatial orientation throughout infancy. According to some authors (e.g., Acredolo, 1990), this egocentric code is gradually replaced by about 14-16 months of age with an allocentric or geocentric representation in which objects and events are coded in terms of important landmarks or environmental features (Acredolo, 1978, 1979; Acredolo \& Evans, 1980; Bai \& Bertenthal, 1992; Bremner, 1978a, 1978b; Bremner \& Bryant, 1977; Goldfield \& Dickerson, 1981; Harris, 1977; Hermer \& Spelke, 1994; Presson \& Ihrig, 1982; Presson \& Somerville, 1985; Rider \& Rieser, 1988; Rieser \& Heiman, 1982; Tyler \& McKenzie, 1990).

This approach assumes that spatial orientation is thus best characterized in terms of the structure of the child's underlying representational system (i.e., egocentric vs. allocentric); such a focus is not the only possibility, however (Corrigan \& Fischer, 1985; Pick \& Lockman, 1981; Presson \& Somerville, 1985). For example, Presson and Somerville discussed a number of conceptual and empirical problems with the idea of representation in general, and egocentrism in particular, and suggested as an alternative that the development of spatial abilities may be better characterized by the uses of spatial information and not by the child's spatial code. These authors distinguished between primary and secondary uses of information, with primary uses involving “. . practical orientation and action in direct relation to aspects of space" (Presson \& Somerville, 1985, p. 15), and secondary uses 
involving the symbolic representation of the environment. Examples of primary actions include, among other behaviors, search for hidden objects, whereas examples of secondary uses include cognitive operations such as mental rotation, drawing maps, and so on.

Within this framework, a critical component in spatial behavior is the orientation task itself and the information available within the environmental context for spatial orientation. Thus, variations in spatial behavior may result from differences in the actual information specifying changes in spatial relations, as well as the subsequent uses of this information. Developmental change, then, may result not from shifting spatial representations but instead from how infants process and use spatial information across task contexts.

This focus on the use of information for spatial orientation and the nature of the task environment raises a variety of questions. Exactly what information is available in the environment for spatial orientation, and how is it described? Is there a systematic relation between the type or amount of information and subsequent spatial behavior? If there are multiple sources of information for spatial relations, what is the relative importance of and relation between these inputs on spatial behavior? And finally, how might the use of this information change as a function of task environment or the age of the perceiver?

As an initial answer to some of these questions, it may be said that under normal circumstances, maintaining spatial orientation is accomplished by means of multiple sources of information. For example, when objects in the world move around us, we become aware of such movements through visual and auditory inputs. When we move through the world, we add kinesthetic, vestibular, and proprioceptive information specifying self movement; for convenience, these latter sources are referred to as body movement information.

What is known concerning children's and adults' use of such information for spatial orientation? Although little work explicitly examines spatial orientation from this framework, a great deal of previous research can be reanalyzed from this point of view (see Presson \& Somerville, 1985, for a review). One such case involves an infant's search for an object or an event following either transformation of the world or movement of the self within the world (Acredolo, 1978, 1979; Acredolo \& Evans, 1980; Bai \& Bertenthal, 1992; Bremner, 1978a, 1978b; Bremner \& Bryant, 1977; Bremner et al., 1994; Butterworth, 1976, 1977; Butterworth, Jarrett, \& Hicks, 1982; Goldfield \& Dickerson, 1981; Laskey, Romano, \& Wenters, 1980). As a classic example, Bremner (1978a, 1978b; Bremner \& Bryant, 1977) investigated an infant's ability to retrieve a toy hidden in one of two locations on a table. Before the child began searching for this toy, the spatial relation between the object and the infant was changed by moving the object relative to the infant (object movement) or moving the infant relative to the object (self movement). In both cases, movements involved $180^{\circ}$ rotations, meaning that a toy originally hidden to the child's left ultimately ended up on the child's right. Bremner (1978a, 1978b) found more accurate search in selfmovement conditions than in object-movement situations, a result that has been replicated in subsequent work (e.g., Bai \& Bertenthal, 1992; but see Goldfield \& Dickerson, 1981, for alternative results).

Although such findings have been interpreted in terms of spatial representation systems, it is also true that the different displace- ments give rise to radically different spatial information. Self movement, for example, produces visual information in the form of optic flow patterns, as well as body movement information, all of which specify a change in position. In contrast, object movement produces visual information for changing spatial relations but no body movement information, and the visual information is markedly different from that produced by movement of the self. One possibility is that it is the availability of the multiple inputs in self movement that leads to more accurate search behavior as opposed to the single input arising from object movement, a possibility raised by Bremner (1978a). ${ }^{1}$

Of course, the idea that the number of inputs specifying changes in spatial relations is related to the efficacy of spatial updating presupposes that all such inputs can be used in spatial orientation. Although it is not controversial to assume that visual information underlies spatial updating, the impact of body movement information is less obvious. There is, however, evidence (McKenzie, 1990; Rider \& Rieser, 1988; Rieser, 1979, 1983, 1989; Rieser, Garing, \& Young, 1994; Rieser, Guth, \& Hill, 1986; Rieser \& Heiman, 1982; Rieser \& Rider, 1991) that body movement information does operate in spatial updating. For example, Rieser et al. (1986), who examined sensitivity to nonvisual information specifying a change in position, found that adults' pointing accuracy to a set of targets was facilitated following blindfolded locomotion to a novel point of observation relative to accuracy following either imagined movement to the same new position or imagined movement combined with walking in a circle; Rieser et al. (1994) extended this finding to children as young as $3 \frac{1}{2}$ years of age. Rider and Rieser (1988), using a different procedure, found that by 2 years of age, toddlers used proprioceptive cues to accurately point to objects in other rooms.

Other studies have looked at younger children. McKenzie (1990), for example, found that 8-month-old infants could be trained to look at a test location even when the site was unmarked by an obvious landmark; this result was presumably due to infants' updating their spatial orientation on the basis of proprioceptive information. Rieser (1979), in the most thorough test of this question, observed that 6-month-olds could use gravitational cues for spatial orientation. In this study, infants received different combinations of visual (dim vs. bright) and vestibular (upright vs. tilted head and body orientation) inputs for a change in position. Following visual manipulations, infants failed to update their spatial orientation; the addition of vestibular information, however, led to increased spatial updating, implying that the infants used gravitational information to keep track of their position in space.

Generally, this work suggests that body movement information can be used for spatial updating, although it does not provide a systematic assessment of the roles of visual and body movement information. The goal of the current project was to provide a factorial combination of information sources, thereby enabling a more thorough test of the number of information sources on spatial orientation. In addition, these studies explored the impact of changing environmental information on search behavior by pro-

\footnotetext{
${ }^{1}$ Both self and object movements contain auditory information as well, although it is not clear how this information relates to the changing spatial relations of the world. Accordingly, this information is not considered further in this article.
} 
viding different cues for the location of a hidden object and comparing the effect of this variation on search.

\section{Experiment 1: Search Following Rotations in Light and Dark-Position Cues}

Experiment 1 represented an initial test of this idea by means of a modified Stage IV search task (Bai \& Bertenthal, 1992; Bremner, 1978a, 1978b; Bremner \& Bryant, 1977; Goldfield \& Dickerson, 1981) in which infants see a toy hidden in one of two locations and then attempt to recover this toy. Before beginning search, infants are either moved around the table (self movement) or the table is moved relative to the infant (object movement); thus, this variable manipulates the presence versus absence of body movement inputs (i.e., vestibular, kinesthetic, and proprioceptive inputs). Visual information is manipulated by either retaining or eliminating the lights within the experimental room. Combining visual information (light vs. dark) and movement type (self vs. object) manipulations thus produces four conditions varying in the information available for spatial updating: object movement in the dark (no on-line source); object movement in the light (single source, visual input); self movement in the dark (single source, body movement input); and self movement in the light (multiple sources, visual and body movement inputs).

This experiment also provided infants with a "position" or "response" cue for coding the location of the object, a cue produced by hiding the toy in the same location across all trials. This environmental cue, adopted from Bai and Bertenthal (1992), allows infants to code the location of the hidden toy in terms of a constant set of motor movements or responses necessary to retrieve the object (Bremner, 1978a, 1978b; Bremner \& Bryant, 1977; Cornell \& Heth, 1979; Newcombe, Huttenlocher, Drummey, $\&$ Wiley, 1998). Such a cue has proven potent for spatial updating in previous research with infants (e.g., Bai \& Bertenthal, 1992; Bremner, 1978a, 1978b; Bremner \& Bryant, 1977; Cornell \& Heth, 1979; McKenzie, 1990).

Finally, this study provided a developmental analysis of the use of multiple inputs in spatial orientation. Previous research has suggested that $8-9$-month-old infants are beginning to use multiple information sources for spatial updating, a finding based on evidence of increased search accuracy in self-movement relative to object-movement conditions (Bai \& Bertenthal, 1992; Bremner, 1978a, 1978b; Bremner \& Bryant, 1977); this result appears firmly established by 14-18 months of age (Acredolo, 1978, 1979; Acredolo \& Evans, 1980). Accordingly, 91/2-18-month-old infants were used in the present study in order to examine the effect of the number of sources of information on orientation with increasing age.

\section{Method}

\section{Participants}

The final sample of participants consisted of 72 children: 24 infants (8 girls) $9 \frac{1}{2}$ months of age $($ mean age $=9.5$ months, range $=9.3-9.7$ months), 24 infants ( 10 girls) 14 months of age (mean age $=14.2$ months, range $=14.0-14.5$ months), and 24 infants (15 girls) 18 months of age (mean age $=18.1$ months, range $=17.9-18.3$ months). An additional nine 91/2-month-old, five 14-month-old, and five 18-month-old infants participated, but their data were not included in this study. Of these infants, 14 were excluded because of fussiness or failure to complete the experiment, 3 because of failure to achieve reliability in search coding (see below), 1 because of experimenter error in running the study, and 1 because his mother kept telling him where to search for the toy. All infants were drawn from the ethnically diverse community of Scarborough, Ontario; detailed demographic information was not collected.

\section{Apparatus}

Infants were tested in a rectangular room $(2.4 \mathrm{~m} \times 1.7 \mathrm{~m})$ formed by hanging orange draperies from ceiling to floor. In the center of this room was a round table ( $51-\mathrm{cm}$ diameter) that sat atop a step stool. Because some trials were to occur in the dark, it was necessary to have children sit on a parent's lap during the study to prevent distress and ensure safety. The parent, in turm, sat on a rolling chair positioned in front of the table, and the experimenter stood directly across the table from infants. A video camera was located outside of the draperies, with its lens positioned through a slit in one drape.

Cups of different colors (height $\approx 15 \mathrm{~cm}$ ) were used to hide objects from infants. During the training phase, a single cup sat in the center of the table, about $19 \mathrm{~cm}$ from the edge of the table near the infant. During the test phase, two cups were placed horizontally in front of the infant, separated by about $19 \mathrm{~cm}$; this distance varied, depending on an infant's size and length of reach. The exact positioning was chosen so that the infant could reach either cup, with the cups spaced far enough apart so that it was difficult to grasp both cups at once. Several small, brightly colored toys were used as the attracting stimuli.

\section{Conditions}

This experiment contained one within-subject and two between-subject manipulations. The within-subject condition involved the type of movement that occurred prior to search. In the self-movement condition the infant was rotated $180^{\circ}$ around the table before beginning search. Self movements were accomplished by pushing the rolling chair containing the parent and child around the table. In the object-movement condition the table was rotated $180^{\circ}$ before the infant searched for the toy. To accomplish object movements, the experimenter lifted the table slightly (to eliminate any noise from movement) and turned the table. As nearly as possible, the time required for self and object movements was the same $(\approx 15-20 \mathrm{~s})$. The first between-subject condition corresponded to the presence versus absence of visual information during self and object movements. In the light condition, movements took place in a normally lit room. In the dark condition, the lights were turned off prior to self-object movements and turned on after the movement, prior to beginning search. The second between-subject manipulation involved the 91/2-, 14, and 18-month-old infants.

\section{Procedure}

Training phase. In this phase, infants learned to search for the toy by means of a procedure used by Bai and Bertenthal (1992). In the first stage, a toy was placed in front of a single cup, and infants were encouraged to retrieve this toy. In the second stage, the toy was partially hidden in the cup, and infants were again encouraged to retrieve the toy. In the final stage, the toy was completely hidden, and infants again retrieved the toy. All infants were required to search successfully twice before moving to the next stage, producing at least six training trials. The purpose of the training phase was to familiarize infants with searching for and retrieving a toy, without biasing for search at a particular location.

All training trials occurred in the following manner. First, the toy was hidden, and infants were rotated $90^{\circ}$ around the table and back; this rotation was in the same direction on training and test trials. The purpose of this $90^{\circ}$ rotation was to familiarize infants with the experience of self movement 
and to try to eliminate any possible distress without requiring spatial updating. Infants in the dark condition had the lights turned off prior to moving, again to familiarize infants to this environmental change. ${ }^{2}$ If infants successfully recovered the toy within $60 \mathrm{~s}$, they were allowed to play with it until the next trial began. If infants failed to recover the toy, it was retrieved by the experimenter. The color of the cup varied across trials to prevent infants from associating a given cup with a specific hiding position, thus rendering cup color irrelevant to object location.

Testing phase. The testing phase began immediately after training. During testing, two cups of different colors (e.g., one red, one blue, although the specific colors changed from trial to trial) were placed on the table, the toy was hidden in one cup, self or object movement occurred, and infants were encouraged to retrieve the toy. If infants successfully found the toy, they were allowed to play with it until the next trial. If infants were unsuccessful, the experimenter revealed the position of the object but did not allow any play with the toy.

Parents held infants in such a way that infants could reach the cups but could not look into the cups, and they also kept infants from retrieving the toy prior to the appropriate movement. Parents were asked not to talk during the experiment if possible, although for infants reluctant to search, parents could encourage toy retrieval. Unfortunately, it was impossible to keep parents naive about the task and the position of the toy: parents were not, however, aware of the experimental hypotheses of this study, nor did they subsequently express awareness of any differences in search across the experimental conditions. Thus, it is unlikely that parents systematically influenced search overall, although if a parent influenced search on a given trial, that trial was immediately rerun.

Infants received four self-movement and four object-movement trials, blocked by condition, and the order of these conditions was counterbalanced across groups of infants. The position cue for object location was provided by consistently hiding the toy in either the left or right cup throughout the experiment (Bai \& Bertenthal, 1992), with side counterbalanced across groups of infants. Half of the children received the light condition, whereas the remaining children received the dark condition. Sessions took approximately $20-30 \mathrm{~min}$ and were videotaped for later reliability coding.

\section{Data Reduction}

In this experiment, a search was defined as any interaction with the cup that revealed its contents: This included lifting the cup, looking in the cup, putting one's fingers inside the cup, knocking the cup over, and so on. Search was initially classified during the session into one of four categories: search in the cup in which the toy was hidden, search in the cup in which the foy was not hidden, search in both cups simultaneously, and failure to search in either cup. Search was then characterized as either correct (1) or incorrect ( 0 ), with correct search defined as searching only in the cup in which the toy was hidden; all other responses were incorrect.

Subsequent to the experiment, a second observer provided reliability coding; reliability was conducted for all sessions for all infants. ${ }^{3}$ Infants with two or more trials on which observers disagreed as to the search behavior, with this disagreement altering the number of correct and incorrect searches, were deemed unreliable. For the $91 \frac{1}{2}-, 14-$, and 18-month-old infants, percent agreements were $99.5 \%, 97.9 \%$, and $97.4 \%$, respectively.

\section{Results and Discussion}

Preliminary analyses examined search as a function of the counterbalanced factors and repeated trial. An initial three-way analysis of variance (ANOVA) was conducted; the betweensubject variables were age ( $91 / 2$ vs. 14 vs. 18 months), order (self movement first vs. object movement first), and side of hiding (left vs. right); this analysis failed to uncover any main effects or interactions (all $F \mathrm{~s}<0.60$ ). A subsequent series of analyses looked at the variable of trial. This analysis was of interest because search on a given trial might be influenced by search on a previous trial in terms of success versus failure in finding the toy, learning effects arising throughout the experiment, or fatigue across trials. To examine these possibilities, 4 two-way ANOVAs were run, with age as the between-subject variable and trial (Trials 1-4) as the within-subject variable; separate ANOVAs were conducted for each light-dark and self-object-movement condition. None of these ANOVAs produced significant effects of trial (all $F$ s $<0.30$ ), and there were no interactions with age, indicating that search did not vary as a function of repeated trial. ${ }^{4}$ On the basis of these effects, all subsequent analyses were collapsed across order, side, and trial variables.

The principal analysis compared search as a function of age, visual information, and movement-type conditions. Given the inherent ambiguity of both and neither responses, which do not truly reflect either absolutely correct or incorrect search, and to facilitate comparing performance with a $50 \%$ chance level, accuracy was based solely on the unambiguous responses of search in the correct or incorrect location. Thus, trials classified as both or neither were removed, with the percent correct in the remaining trials then calculated.

The data were analyzed in a three-way ANOVA, with the between-subject variables of age ( $91 / 2$ vs. 14 vs. 18 months) and visual information (light vs. dark environment) and the withinsubject variable of movement type (self vs. object movement). Of the three main effects, there was a significant difference for movement type, with better search following self movement $(M=48.2 \%, S D=37.4)$ relative to object movement $(M=37.2 \%, S D=34.9), F(1,65)=5.48, M S E=0.08, p<.05$. There was no effect for visual information, $F(1,65)=1.29, M S E$ $=0.19(n s)$, or age, $F(2,65)=0.14, M S E=0.19(n s)$. The only other significant result was the critical interaction between visual information and movement type, $F(1,65)=5.94, M S E=0.08$, $p<.05$. This interaction (see Figure 1) revealed that search was better when self movement occurred in the light; all other condi-

\footnotetext{
${ }^{2}$ Although creating a systematic difference in training between the two conditions, this procedure does retain consistency within the visual environment between training and test trials. The fact that all infants had to reach a common criteria in training ensures that they learned the game of retrieving the toy equally well. Thus, differences as a function of visual environment cannot be attributed to differential effectiveness in initial training.

${ }^{3}$ For one $91 / 2$ - and one 14-month-old infant, one trial during the session was not recorded on the videotape; the remainder of trials for both infants, however, were reliable.

${ }^{4}$ It is also possible to conduct an omnibus ANOVA including trial and side factors along with the other experimental variables. In fact, such an analysis was conducted for this and the remaining experiments and consistently failed to reveal any interactions between trial and side factors and the remaining variables. Because these analyses overlap substantially with the principal analyses for these experiments, these results are not presented separately.
} 


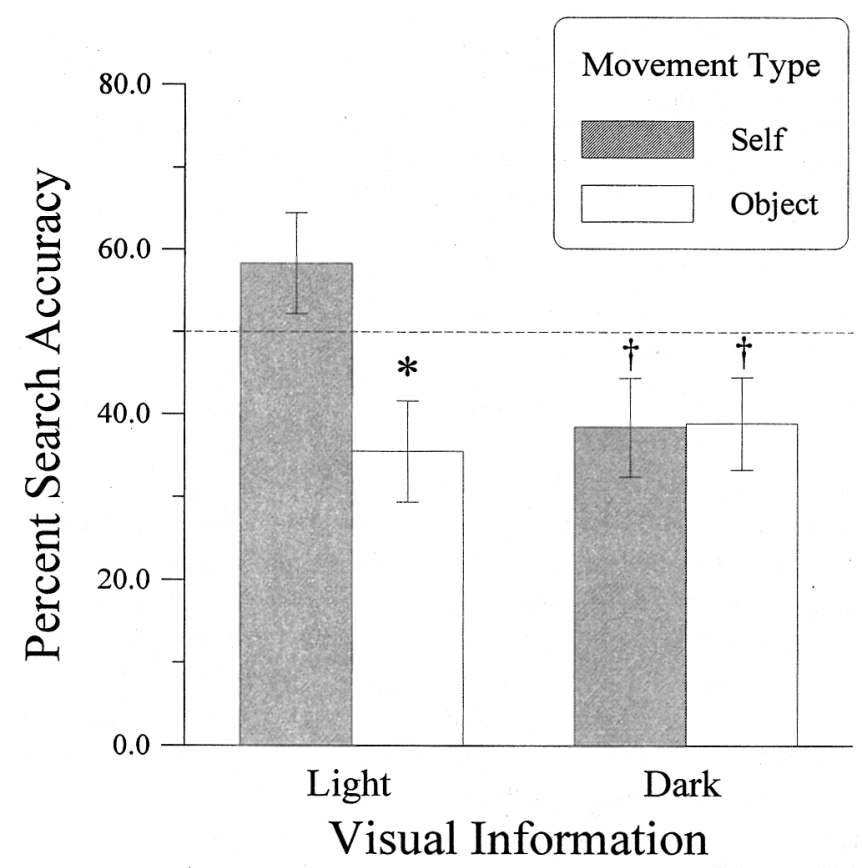

Figure 1. The mean percentage search accuracy as a function of the movement type and visual information conditions for Experiment 1. Chance responding is shown in the figure by the dashed line; accuracy differing significantly from chance is also noted. ${ }^{\dagger} p \leq .06 .{ }^{*} p<.05$.

tions led to equivalently worse performance. ${ }^{5}$ Although a null effect, it is noteworthy that age did not influence search overall (see above) or in interaction with visual information and movement type, $F(2,65)=0.42, M S E=0.08(n s)$. Thus, older infants were no more adept than younger infants at finding a hidden toy or at using either visual or body movement inputs for updating.

Despite the preliminary analyses demonstrating no significant effects associated with trial, a comparable three-way ANOVA looked at search accuracy for the first (after removal of both and neither responses) trial. The purpose of this analysis was twofold: to provide a point of comparison with other published work that restricted analyses to first trial data (e.g., Bai \& Bertenthal, 1992) and to determine whether the pattern of results just described similarly holds for trials that are "uninfluenced" by previous responses. The mean accuracies for the visual and body movement conditions, collapsed across age, are shown in Table 1 and reveal a pattem comparable to that seen in the analysis for all of the trials; search following self movement in the light was more accurate than search in the remaining three conditions. Although this interaction failed to achieve statistical significance, it should be remembered that first trials represent only slightly more than one fourth of the data set overall, and thus a greater degree of variability is to be expected. ${ }^{6}$ As such, the critical point is that the same general pattern emerges from this analysis as in the previous analysis for all of the trials. Viewed in this vein, this analysis complements the earlier results that search did not vary as a function of repeated trial.

A final series of analyses compared search to chance responding. Averaging across age, $50 \%$ was subtracted from search accuracy and, in a series of two-tailed $t$ tests, the resulting difference scores were compared with zero. These findings also appear in Figure 1 and reveal that accuracy following self movement in the light did not differ significantly from $50 \%, t(34)=1.35$ (ns), whereas the remaining conditions produced accuracy less than chance: $t(34)=2.37, p<.05$ (object movement in the light), $t(35)=1.92, p=.06$ (self movement in the dark), and $t(35)=1.96, p<.06$ (object movement in the dark). One possible interpretation of this result is that self movement in the light induced better spatial orientation than the remaining three conditions. Conceptually, though, it is difficult to accept the claim that infants truly updated their position given that they did not, in fact, recover the toy more than predicted by chance; for this argument to be compelling, one would hope to see above-chance accuracy. On the basis of the significant Visual $\times$ Movement Type interaction, one can claim that self movement in the light produced search that was less egocentric than the remaining conditions and that search was clearly influenced by the information available in this condition; the exact nature of this influence is ambiguous, however.

In sum, this study replicated the finding of better search following self movement relative to object movement in a lit environment (Bai \& Bertenthal, 1992; Bremner, 1978a, 1978b; Bremner \& Bryant, 1977). What is new in this study is the systematic comparison of visual information and movement type and the finding that object movement in the dark, self movement in the dark, and object movement in the light produced significantly inaccurate (egocentric) search, whereas self movement in the light led to search at chance (less egocentric) levels. Although the interpretation of this difference remains ambiguous, these results do suggest that neither visual nor movement-type information by itself produces better spatial updating.

\section{Experiment 2: Search Following Rotations in Light and Dark-Color Cues}

Experiment 2 was intended to again assess the impact on search of systematic combinations of visual information and movement type in the context of providing a stronger environmental cue for object location. Specifically, this study coded the correct location with a color cue, produced by consistently hiding the toy in a single colored cup across trials. Previous work (Bremner, 1978a, 1978b; Cornell, 1981) has shown that color information provides an extremely powerful cue for coding hidden object location.

Providing a more potent cue enabled evaluation of the impact of environmental cue structure on search through comparison with

\footnotetext{
${ }^{5}$ Both and neither categories accounted for $5.90 \%$ of the data $(5.03 \%$ and $0.87 \%$, respectively). Unfortunately, once these responses were removed, one $91 / 2$-month-old infant in the light condition had no usable trials in one condition, and this infant's data were thus dropped from the analys. To ensure that analyzing only unambiguous responses did not substantively alter the results, an analysis was conducted in which the entire data set (e.g., including both and neither responses) was used. This analysis produced equivalent findings, with a single main effect of movement type and a significant Visual Information $\times$ Movement Type interaction.

${ }^{6}$ The reason that first trials did not account for exactly one fourth of the entire data set is that some trials were removed because they were coded as both or neither.
} 
Table 1

Mean Percent Correct on First Trials, Averaged Across Age, as a Function of Visual Information and Movement-Type Conditions: Experiments 1, 2, and 3

\begin{tabular}{|c|c|c|c|c|c|c|}
\hline \multirow[b]{2}{*}{ Type of movement } & \multicolumn{2}{|c|}{$\begin{array}{l}\text { Experiment } 1 \\
\text { (position cues) }\end{array}$} & \multicolumn{2}{|c|}{$\begin{array}{c}\text { Experiment } 2 \\
\text { (color cues) }\end{array}$} & \multicolumn{2}{|c|}{$\begin{array}{c}\text { Experiment } 3 \\
\text { (position \& color cues) }\end{array}$} \\
\hline & $\begin{array}{c}\text { Light } \\
(n=35)\end{array}$ & $\begin{array}{c}\text { Dark } \\
(n=36)\end{array}$ & $\underset{(n=39)}{\text { Light }}$ & $\begin{array}{c}\text { Dark } \\
(n=40)\end{array}$ & $\begin{array}{c}\text { Light } \\
(n=39)\end{array}$ & $\begin{array}{c}\text { Dark } \\
(n=40)\end{array}$ \\
\hline Self & 57.1 & 38.9 & 61.5 & 50.0 & 56.4 & 52.5 \\
\hline Object & 40.0 & 41.7 & 53.8 & 60.0 & 51.3 & 55.0 \\
\hline
\end{tabular}

Experiment 1, as well as resolution of the ambiguity of interpretation highlighted earlier. If self movement in the light truly does produce better orientation, then this condition should continue to lead to enhanced accuracy even when search is more accurate overall. If, however, this condition actually produces random search (through, say, confusion of the child), which then looks like better performance relative to the egocentric responding in the remaining conditions, then accuracy following self movement in the light should remain at chance levels.

Finally, this study provided another opportunity to assess age differences in the use of visual information and movement type. To simplify the design, 91/2- and 16-month-old infants were examined; these ages were chosen to straddle a range that undergoes significant change in spatial abilities (Acredolo, 1978, 1979; Acredolo \& Evans, 1980).

\section{Method}

\section{Participants}

The final sample of participants consisted of 80 children: 40 infants (22 boys) $9 \frac{1}{2}$ months of age ( $M$ age $=9.5$ months, range $=9.0-9.7$ months) and 40 infants ( 18 boys) 16 months of age ( $M$ age $=15.9$ months, range $=15.2-16.2$ months). An additional six $91 / 2$-month-old infants and ten 16-month-old infants participated, but their data were not included in this study. Of these infants, 12 were excluded because of fussiness, 2 because of problems with the video equipment, 1 because he was more than 4 weeks premature, and 1 because data coding proved unreliable. All infants were drawn from the culturally diverse Scarborough, Ontario, community.

\section{Apparatus, Conditions, Procedure, and Data Reduction}

The apparatus and conditions of this study were the same as those of Experiment 1 except that the room size was increased to $2.4 \mathrm{~m} \times 2.3 \mathrm{~m}$ to allow easier passage around the table. The principal procedural change was in the test phase, in which the cup colors for the correct and incorrect locations remained constant across trials. For each infant, two cup colors were chosen; the cup that was to contain the toy was picked at random but held constant for each infant. Left-right position of the hidden toy was randomized across trials: The toy was placed on the left side half of the time in each condition and on the right in the remaining half.'

The protocols for training and test phases, as well as the data coding and reduction, were identical to those of Experiment 1. Subsequent coding of search by a second observer revealed high reliability, with percent agreements of $99.4 \%$ for $91 / 2$-month-olds and $99.1 \%$ for 16 -month-olds.

\section{Results and Discussion}

Using the entire data set, preliminary analyses examined search as a function of the counterbalanced variables and repeated trial.
An initial three-way ANOVA failed to reveal an effect of order (self movement first vs. object movement first), $F(1,76)=0.20$, $M S E=0.06(n s)$, although there was a main effect of side of hiding (left vs. right), $F(1,76)=6.36, M S E=0.11, p<.05$; accuracy was greater when the toy was on the left $(M=55.9 \%, S D$ $=29.6)$ than on the right $(M=42.7 \%, S D=28.1)$. No other effects were significant. Subsequent two-way ANOVAs examined search across repeated trial for each visual and movement-type condition individually. Of the four ANOVAs, the only significant result was a main effect of trial for self movement in the dark, $F(3$, 114) $=3.13, M S E=0.26, p<.05$, with performance on the second trial worse than on the remaining trials; none of the conditions produced monotonically increasing or decreasing search accuracy, however. Given these findings, all data were collapsed across these variables in subsequent analyses.

The principal analyses involved a three-way ANOVA using the unambiguous measures of accuracy described earlier, with the between-subject variables of age ( $91 / 2$ vs. 16 months) and visual information (light vs. dark) and the within-subject variable of movement type (self vs. object movement). This analysis revealed a main effect of visual information, with search in the light $(M=61.3 \%, S D=27.1)$ exceeding search in the dark $(M=48.1$, $S D=20.9), F(1,75)=11.77, M S E=0.06, p<.001$. Most important was the significant interaction between visual information and movement type, $F(1,75)=7.03, M S E=0.05, p<.01$, which was a replication of the interaction in Experiment 1; this interaction (see Figure 2) again revealed better search for self movement in the light than the remaining three conditions. The three-way interaction between these variables was not significant, $F(1,75)=0.30, M S E=0.05(n s)$. These results confirm the finding that for both ages, visual information and movement type are necessary for more accurate spatial updating. ${ }^{8}$

As in Experiment 1, a comparable analysis using only the data from the first usable trial was conducted. Presented in Table 1, these means display a pattern similar to that found for data from all trials, with maximum performance following self movement in the

\footnotetext{
${ }^{7}$ One infant in one condition received three trials on the left and one trial on the right.

${ }^{8}$ As in Experiment 1, both and neither categories accounted for $9.53 \%$ ( $8.65 \%$ and $0.78 \%$, respectively) of responses; excluding these trials meant that one 16-month-old infant in the light condition had to be removed because of missing data. Once again, an ANOVA using the entire data set produced results equivalent to those reported in the text; the only noteworthy difference was a marginal effect of age, $F(1,76)=3.87, M S E=0.21$, $p<.06$, with $91 / 2$-month-olds outperforming 16-month-olds.
} 


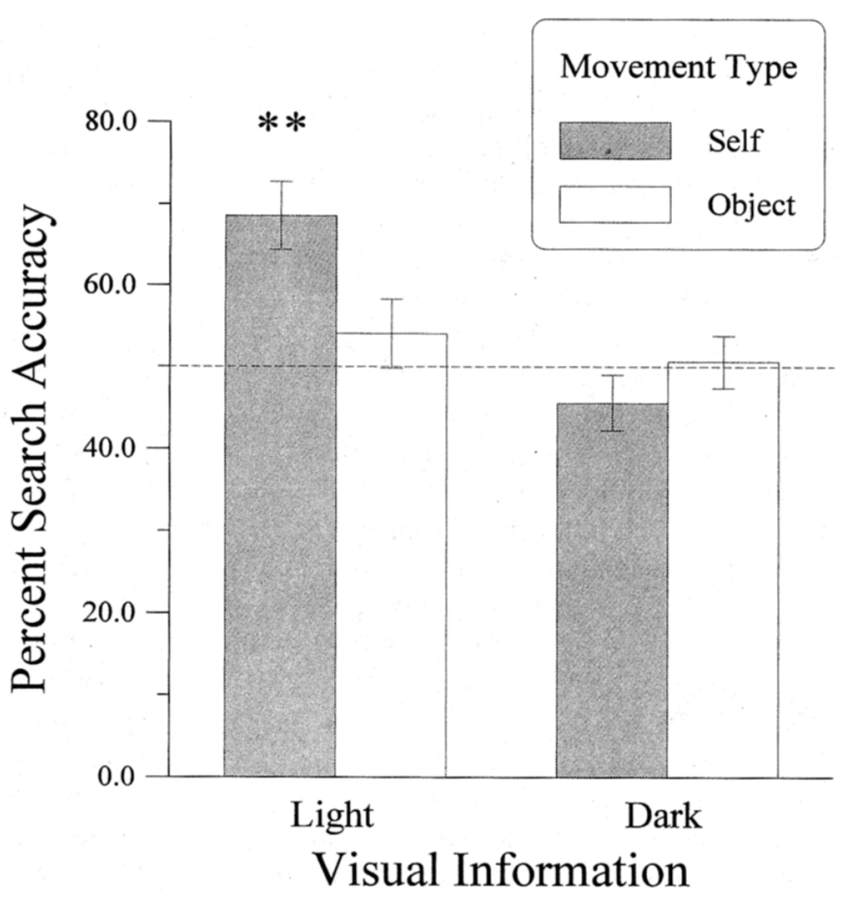

Figure 2. The mean percentage search accuracy as a function of the movement type and visual information conditions for Experiment 2. Chance accuracy is shown in the figure by the dashed line; accuracy differing significantly from chance is also noted. ${ }^{* *} p<.01$.

light. Although this analysis again failed to reveal any significant differences, recall that first trials account for only about one fourth of the entire set; hence, these values should be more variable.

Finally, search behavior was assessed relative to chance by testing difference scores for each condition against zero. The results of these analyses also appear in Figure 2 and demonstrate that self movement in the light led to accuracy exceeding chance, $t(38)=4.59, p<.001$, whereas the remaining conditions led to search at or below chance levels.

In sum, Experiment 2 found increased accuracy in infants' searching for a hidden toy following self movement in the light relative to all other situations; this condition produced search that was significantly better than expected by chance. In contrast, object movement in the light and self and object movements in the dark produced search at or below chance levels. One implication of these results is that self movement in the light truly produced better spatial orientation than did the remaining conditions and, by analogy, suggests that the increased accuracy for this condition in Experiment 1 similarly reflected better spatial updating. Although resolving the earlier ambiguity, this speculation represents, admittedly, an interpretative leap.

This study also found that using a color environmental cue produced better search than did the position cue of Experiment 1, with this difference between color and position environmental cues adding to the impact on search produced by combining visual information and movement type. It is important to note, though, that these studies actually placed color and position cues into conflict. In Experiment 1, because the target was specified by means of a constant position cue across trials, any cue based on cup color was at best inconsistent and at worst misleading. In contrast, in Experiment 2, when the color cue consistently indicated target position, left-right position information was unreliable. This conflict between the cues raises the question of what would happen if color and position cues concurrently specified the correct hiding position; this possibility is examined in Experiment 3.

\section{Experiment 3: Search Following Rotations in Light and Dark-Combined Color and Position Cues}

In Experiment 3, search following self and object movements in the light and dark was again examined. This experiment was different from the previous two in that it combined the environmental cues used earlier. Thus, for each infant, the toy was hidden in a particular colored cup, on the same side of the table on every trial. In all other respects this study duplicated the previous experiments.

\section{Method}

\section{Participants}

The final sample of participants consisted of 80 children: 40 infants (19 girls) $91 / 2$ months of age (mean age $=9.5$ months, range $=9.1-9.8$ months) and 40 infants ( 24 girls) 16 months of age (mean age $=16.0$ months, range $=15.6-16.9$ months). An additional ten 91/2-month-old infants and seven 16-month-old infants participated, but their data were not included in this study. Of these infants, 15 were excluded because of fussiness during the experiment, 1 because he/she was 5 weeks or more premature, and 1 because of equipment failure during the experiment.

\section{Apparatus, Conditions, Procedure, and Data Reduction}

The sole procedural change in this study involved the testing phase, in which the correct location of the hidden toy was marked by both a consistent cup color and a consistent side of hiding. So, for example, on all self- and object-movement trials, infants would see the toy hidden in a red cup, on the left side of the table; specific cup colors were chosen randomly for each infant. The conditions, experimental protocol, coding, and data reduction were all identical to those of Experiments 1 and 2. Subsequent coding indicated that search behavior was highly reliable, with percent agreements of $100 \%$ and $99.7 \%$ for $91 / 2$ - and 16 -month-old infants, respectively.

\section{Results and Discussion}

Using the data from all triais, an initial ANOVA was conducted in order to examine search as a function of age ( $91 / 2 \mathrm{vs.} 16$ months), order (self movement first vs. object movement first), and side of hiding (left vs. right). Of the effects involving the counterbalanced factors (age is discussed below), this analysis revealed a significant three-way interaction, $F(1,72)=5.97, M S E=0.80, p<.05$, with roughly equal performance across order and sides for 16-montholds, whereas $91 / 2$-month-olds varied (uninterpretably) across these variables. Subsequent two-way ANOVAs, using age and trial factors, found no effects of trial (all $p s>.30$ ) nor any interactions between the two (all $p s>.50$ ). Consequently, all data were collapsed across these factors.

As before, the principal analysis used the unambiguous data set and compared search as a function of age (91/2 vs. 16 months), visual information (light vs. dark), and movement type (self vs. 
object movement). This analysis produced marginally significant effects of age, $F(1,75)=3.20, M S E=0.15, p=.08$ and visual information, $F(1,75)=2.95, M S E=0.14, p=.09$. There was also a marginally significant interaction between age and visual information, $F(1,75)=3.75, M S E=0.14, p=.06$, with $91 / 2-$ month-olds showing equivalent performance in light and dark conditions, whereas 16-month-olds demonstrated better search in the light than in the dark. Most important, however, was the interaction between visual information and movement type, $F(1$, $75)=2.88, M S E=0.08, p=.09$. Although only marginally significant, the pattern of responding was similar to that of the previous two studies (see Figure 3); search following self movement in the light was greater than in the other three conditions. Comparing accuracy to chance revealed that search following self movement in the light was greater than chance, $t(38)=3.07, p<$ .005 ; none of the remaining conditions produced search varying from chance performance. ${ }^{9}$

As with the previous experiments, an analysis restricted to the first trial data produced a comparable pattern of results, with maximum accuracy following self movements in the light (see Table 1). Once again, because of the increased variability resulting from analyzing only a subset of the data, this analysis failed to reveal any significant effects.

\section{Cross-Experiment Comparisons}

In the final set of analyses, search across the three experiments was compared using the unambiguous data set (i.e., after removing both and neither responses) in a four-way ANOVA, with the

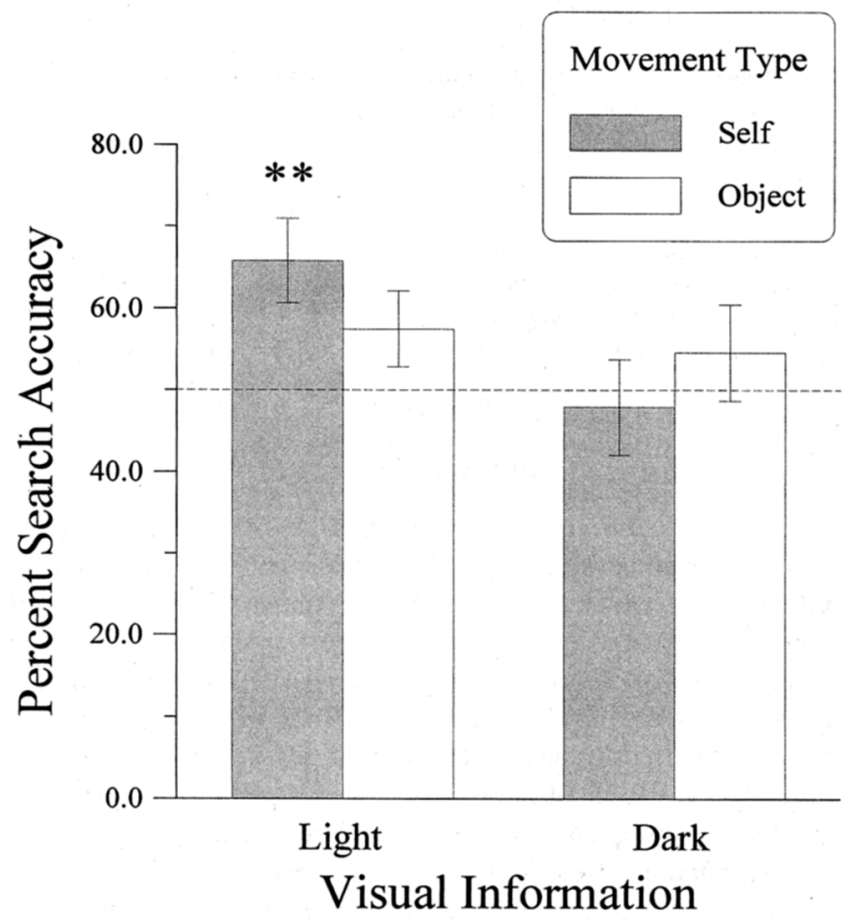

Figure 3. The mean percentage search accuracy as a function of the movement type and visual information conditions for Experiment 3. Chance accuracy is shown in the figure by the dashed line; accuracy differing significantly from chance is also noted. ${ }^{* * *} p<.01$. between-subject factors of experiment (Experiment 1 vs. Experiment 2 vs. Experiment 3), visual information (light vs. dark), and age group (young vs. old) and the within-subject factor of movement type (self vs. object movement). For the age group variable, the $91 / 2$-month-olds in all three experiments were designated the young group, whereas the 14- and 18-month-olds (Experiment 1) and the 16-month-olds (Experiments 2 and 3) were designated the old group. This ANOVA revealed three main effects. First, there was an effect of experiment, $F(2,217)=5.91, M S E=0.13, p<$ .005 , with average accuracy in Experiments 2 and $3(\mathrm{Ms}=54.6 \%$ and $56.4 \%, S D_{\mathrm{S}}=25.0$ and 34.3 , respectively) exceeding that in Experiment $1(M=42.7 \%, S D=36.5)$. Second, there was an effect of visual information, $F(1,217)=9.51, M S E=0.13, p<$ .005 , with search following movements in the light $(M=57.0 \%$, $S D=32.6$ ) exceeding search following movements in the dark $(M=46.3 \%, S D=31.9)$. Third, there was an effect of movement type, $F(1,217)=4.74, M S E=0.07, p<.05$, with self movement producing more accurate search than object movement $(M \mathrm{~s}=54.2 \%$ and $48.9 \%, S D \mathrm{~s}=33.4$ and 31.7 , respectively $)$. Along with a marginal interaction between age and visual information, $F(1,217)=3.08, M S E=0.13, p=.08$, with the difference between light and dark conditions more pronounced for older than for younger infants, the only other significant result was the interaction between visual information and movement type, $F(1,217)=15.34, M S E=0.07, p<.001$. The form of this interaction was identical to that for all three studies, with search following self movement in the light $(M=64.4 \%, S D=31.7)$ significantly exceeding chance, $t(112)=4.85, p<.001$, and the remaining conditions producing search less than or equivalent to chance. The three-way interaction between experiment, visual information, and movement type was not significant, $F(2$, $217)=0.82, M S E=0.13(n s)$, suggesting that the Visual $\times$ Movement interaction characterized all three experiments.

In sum, the findings of Experiment 3 confirmed those of the previous studies in demonstrating that self movement in the light led to more accurate search than did object movement in the light, self movement in the dark, and object movement in the dark; these latter three conditions produced approximately equivalent search. Although this finding is tempered somewhat by the marginal interaction between these variables, the fact that the form of this interaction was identical to that of the previous studies, the findings relative to chance, and the lack of differences between the experiments in terms of this interaction combined to bolster this effect.

Experiment 3 went beyond the previous studies in finding that a simultaneous color and position cue for the correct location did not increase performance relative to accuracy with only a color cue. It is possible that this result is due to a ceiling effect, although even

\footnotetext{
${ }^{9}$ Both and neither categories accounted for $5.5 \%$ of the data $(3.1 \%$ and $2.4 \%$, respectively); removal of these responses necessitated dropping one $91 / 2$-month-old in the light condition. A three-way ANOVA on the entire set produced results equivalent to those reported in the text; the sole difference was a significant main effect of age, $F(1,76)=4.00$, $M S E=0.15, p<.05$, with $91 / 2$-month-olds again outperforming 16-montholds. It is intriguing that in both Experiments 2 and 3, the difference between the two ages was reduced or disappeared when a more conservative analysis criterion was used, which might imply the use of a qualitatively different response strategy in older infants.
} 
if one accepts a functional ceiling of $65-70 \%$, this does not explain why redundant environmental cues failed to boost performance in the remaining conditions, which were below this level.

\section{General Discussion}

The principal goal of these studies was to examine whether spatial orientation could be characterized by information underlying spatial behavior, as opposed to the structure of one's representational code for spatial relations (Presson \& Somerville, 1985). To explore this idea, the information available for on-line spatial updating (visual vs. body movement information) was manipulated, along with the environmental information specifying the location of the hidden object (position vs. color cue). Overall, these studies convincingly demonstrate that spatial behavior can be described in terms of the spatial information present. For example, three experiments demonstrated that search accuracy improved with increasing amounts of information specifying a change in spatial relations. Thus, multiple sources of information, produced by self movement in the light, consistently led to more accurate search than did only a single source of information, as occurs with object movement in the light and self movement in the dark. The impact of the amount of information was not simply additive, however. The two single-input conditions resulted in no better search than having no on-line, continuous information for updating, as produced by object movement in the dark. Such results strongly suggest that visual and body movement information interact in search behavior.

There are two primary findings arising from these studies that beg explanation. First, there is the fact that in all three experiments, search following self movement in the light exceeded search in the remaining conditions. Although this result has been attributed to the idea that there is more information available for spatial orientation in this condition, no real attempt has been made to describe the nature of this information or the underlying processes that use this information and produce the observed differences between conditions. Second, there is the result that the color cue, available in Experiments 2 and 3, produced significantly better search than the position cue available in Experiment 1. Again, little has been said concerning the processes that may underlie this difference in search behavior.

As for explaining the differences in search accuracy between self and object movements in the light and dark, some insight may be gained through considering the information available in these conditions and its implications for spatial coding and updating. For example, self movement in the light produces global optical flow information that strongly and effectively signals to observers a change in position in the world (via visual proprioception; Gibson, 1966; Lee \& Aronson, 1974; Lee \& Lishman, 1977; Lishman \& Lee, 1973) and specifies the actual direction in which one is heading (e.g., Cutting, 1986; Lee, 1993; Warren \& Hannon, 1988; Warren, Mestre, Blackwell, \& Morris, 1991; Warren, Morris, \& Kalish, 1988). This percept of self movement is reinforced by the body movement-primarily vestibular system-information that similarly signals a change in position as well as a rotational path through the world. That neither of these inputs requires focused attention to specific features of the environment means that there will always be compelling information signaling a change in position and thereby allowing for on-line spatial updating.
This inescapable information specifying a change in position, and hence in spatial relations in the world, may result in better spatial updating through the processes of (a) increasing visual attentiveness toward the location of the hidden toy and (b) inducing more updating of one's spatial orientation toward the toy, perhaps using a more objective spatial code. Put differently, the unavoidable awareness that they are moving causes infants to pay more attention to their surroundings and their relation to the location of objects in the world; such increased attentiveness could easily produce less egocentric spatial coding.

Obviously, increased visual attentiveness toward the location of the hidden toy is not an option when spatial changes take place in the dark. Thus, although self movement in this situation provides compelling signals for a change in position and may thus lead infants to attempt to increase their visual attentiveness and spatial orienting, such updating cannot occur (on the basis of visual input) during the actual movement. Instead, for self and object movements in the dark, updating must be accomplished after the change has occurred and will thus depend heavily on noticing external environmental cues for how the world has changed, thereby potentially reducing search accuracy in the dark as opposed to the light conditions. Although visual attentiveness toward the target location is possible during object movement in the light, the local optic flow in this condition is limited to specifying a change in object position (as opposed to position of the self) and is dependent upon focused attention toward a particular location in space within a circumscribed period of time. It is possible that an observer, and especially an infant, may simply miss this critical information regarding how the world changes. Thus, as with the two dark conditions, updating of the spatial location of the hidden object is reliant on the use of external environmental cues.

Support for this argument comes from two sources. First, there is ample evidence that increased visual attentiveness to object or event location significantly facilitates spatial orientation and updating (Acredolo, 1985; Acredolo, Adams, \& Goodwyn, 1984; Bai \& Bertenthal, 1992; Gratch \& Landers, 1971; Gratch et al., 1974; Horobin \& Acredolo, 1986). Second, there is evidence (albeit indirect) that attentiveness may vary as a function of self versus object movement. Bai and Bertenthal, for example, found a stronger relation between search accuracy and visual tracking for self, as opposed to object movement conditions (see Bai \& Bertenthal, 1992 , p. 221), although both correlations were statistically significant. Because the current experimental setup did not allow for a systematic assessment of visual tracking in these studies, it is not possible to directly test this hypothesis with these data. Ongoing research is currently exploring this possibility.

As for the finding that the color cue led to greater search accuracy than the position cue, one possible explanation involves the nature of the underlying process used by infants to code and remember the position of the object prior to and following self and object movement. For the color cue, the code used to mark the toy location before the movement (e.g., "the toy is in the red cup") produces correct search following self-object movement ("the toy is still in the red cup"). In contrast, the position cue before self-object movement (e.g., "the toy is in the right cup"), unless updated, produces incorrect search following movement ("the toy is now in the left cup"). Accordingly, the efficacy of this cue will thus depend quite heavily on information available to infants 
suggesting the need for, and how to, update their spatial position; this is the type of information that has just been described.

A second possibility is that color is a more distinct cue, one that is directly correlated with target position (e.g., "in the red cup"). In contrast, although position can code object location directly (e.g., "in the right cup"), which then requires updating after movement (see above), it may also provide a less distinct, more weakly correlated cue for object location (e.g., "located to the right"). Given that numerous researchers have observed better search accuracy when object location is coded by more, as opposed to less, distinctive cues (Bremner, 1978a, 1978b; Bushnell, McKenzie, Lawrence, \& Connell, 1995; Butterworth et al., 1982; Comell, 1981), this latter coding will thus produce poorer performance.

A different concern with these studies is that the level of overall search accuracy across all experiments was not especially high; the average best case performance in any condition was approximately $67 \%$ correct. Such accuracy is troubling in that it raises the specter of a procedural problem with the experimental situation. Although this is a serious consideration, it is worth emphasizing that the absolute level of performance in these studies is of only subsidiary importance to the experimentally and theoretically critical finding of differential performance between the conditions. In this regard, the crucial result is that search following self movement in the light exceeded search in the remaining conditions; this pattern was replicated across multiple experiments with varying performance levels and differing environmental contexts.

In this regard, it is critical to note that these studies presented an especially difficult orientation situation for infants. For example, these studies used an unfamiliar room devoid of obvious landmarks useful in establishing an external reference frame; both unfamiliarity and the lack of obvious landmarks have demonstrably negative effects on search (Acredolo, 1979; Acredolo \& Evans, 1980; Bushnell et al., 1995; Hermer \& Spelke, 1994; Presson \& Ihrig, 1982; Rieser, 1979). Moreover, the transformations involved in these studies were also difficult. Moving infants $180^{\circ}$ around a table necessitates maintaining spatial orientation during a combined rotation and translation through the world; object movements required keeping track of invisible object displacements. Previous research has also found that these factors are particularly difficult for infants to handle (e.g., Landau \& Spelke, 1985, 1988), and as such, it is not surprising that even the best case performance was not optimum.

Finally, and most significantly, it is not clear that the level of search accuracy in these studies is particularly deviant with respect to the literature on spatial orientation. Over the course of three experiments, accuracy varied from about $30-35 \%$ to $65-70 \%$ correct. In fact, meta-analyses looking at probability correct in search tasks (e.g., Marcovitch \& Zelazo, 1999; Wellman, Cross, \& Bartsch, 1986) suggest that the accuracy in such studies typically falls exactly within this range.

As an example, Bremner and Bryant (1977) reported the number of 9-month-old infants who searched perseveratively for a hidden toy, following either self or object movement. For the present purposes, the most relevant results are for infants in Groups $A$ and C, reported in Bremner and Bryant's Table 3 (p. 169), who were in conditions comparable to the self- and object-movement conditions (in the light) of the current experiments. Across five trials, infants in Bremner and Bryant's study erred in search on 40 and 46 of 80 trials, for accuracies of $50 \%$ and $42.5 \%$ in self- and object- movement conditions, respectively; such accuracy is remarkably similar to Experiment 1 of the present study. Similarly, Bremner (1978a) looked at 9-month-old infants' search following self and object movement when toy position was marked by distinctive background as opposed to cover cues. In this study, Groups B and D (background and cover cues, respectively) were equivalent to the current self movement in the light condition, whereas Groups $\mathrm{C}$ and $\mathrm{E}$ (background and cover cues, respectively) were equivalent to the object-movement condition. On the basis of the mean error across five trials shown in Bremner's (1978a, p. 353) Table 2, infants in the self-movement conditions were $63.8 \%$ and $73.8 \%$ correct (background and cover), whereas object movement produced accuracies of $40.0 \%$ and $50.0 \%$. Although the accuracies for the self-movement conditions (Groups B and D) were slightly higher than those observed in the present studies, Bremner (1978a) did conduct his study in the parent's home, an environment typically observed to lead to better spatial orientation than laboratory studies (e.g., Acredolo, 1979). Finally, Bai and Bertenthal (1992) examined 8-month-old infants' search following self and object movement. Although these authors only reported the number of infants searching correctly on the first of five trials (Bai \& Bertenthal, 1992, Table 2, p. 220), the percent correct responses for the most motorically advanced group (creeping infants) were $72.2 \%$ and $55.6 \%$ for self- and object-movement conditions, respectively.

Generally, numerous studies using manual search tasks (Acredolo, 1979; Acredolo et al., 1984; Bai \& Bertenthal, 1992; Bjork \& Cummings, 1984; Bremner, 1978a, 1978b; Bremner \& Bryant, 1977; Bremner et al., 1994; Butterworth, 1977; Butterworth et al., 1982; Cornell, 1981; Goldfield \& Dickerson, 1981; Sophian \& Wellman, 1983) and event anticipation (e.g., Acredolo, 1978; Acredolo \& Evans, 1980; Bertenthal, Campos, \& Barrett, 1984; Rieser, 1979) have observed that accuracy typically varies within the $25-30 \%$ to $75-80 \%$ range; overall, this is very much in line with the current data. One reason the present experiments might, at first blush, seem unusual is that few studies actually report accuracy as such. Instead, they typically present either the number of errors (summed or average) made by infants, or the number of infants who respond egocentrically; true accuracy, in terms of percent correct, is seldorn explicitly given.

This article has focused on the different types of information that are available to guide the infant's spatial orientation rather than on how that information is represented in a spatial code. Unfortunately, whether spatial orientation (a) involves a shift from egocentric to allocentric coding within the first few years (e.g., Acredolo, 1990), (b) is primarily egocentric (e.g., Bremner et al., 1994), or (c) involves coordinating both representations (e.g., Harris, 1977; Millar, 1993) cannot be addressed by the present studies. It seems clear that, in at least some conditions of the present experiments, spatial coding was egocentric (i.e., systematic below-chance responding); whether this conceptualization best captures infants' spatial abilities per se is a different question.

Finally, one of the more puzzling findings of these experiments was the paucity of differences between the ages. This lack of age differences raises the question of when, developmentally, infants might begin to use partial information conditions to orient as successfully as they do using multiple sources of information. Unfortunately, the answer to this question is also unknown. Expanding the range of ages examined, as well as employing an 
easier task with an environment containing more cues, might well reveal developmental differences and progressions in this regard.

In sum, the present studies investigated the role of visual and body movement information on spatial orientation and updating. One conclusion arising from this work is that orientation can be characterized by the availability of information for spatial updating and object localization, findings consistent with ideas expressed by Presson and Somerville (1985). Although compelling, these results are only a first step in exploring these ideas. Subsequent research should further explore the role of visual, auditory, and motor information, the impact of environmental cues on spatial orientation, as well as ways to extend this approach to other spatial paradigms.

\section{References}

Acredolo, L. P. (1978). Development of spatial orientation in infancy. Developmental Psychology, 14, 224-234.

Acredolo, L. P. (1979). Laboratory versus home: The effect of environment on the 9-month-old infant's choice of spatial reference system. Developmental Psychology, 15, 666-667.

Acredolo, L. P. (1985). Coordinating perspectives on infant spatial orientation. In R. Cohen (Ed.), The development of spatial cognition (pp. 115-140). Hillsdale, NJ: Erlbaum.

Acredolo, L. P. (1990). Behavioral approaches to spatial orientation in infancy. In A. Diamond (Ed.), Annals of the New York Academy of Sciences: Vol. 608. The development and neural bases of higher cognitive functions (pp. 596-607). New York: New York Academy of Sciences.

Acredolo, L. P., Adams, A., \& Goodwyn, S. W. (1984). The role of self-produced movement and visual tracking in infant spatial orientation. Journal of Experimental Child Psychology, 38, 312-327.

Acredolo, L. P., \& Evans, D. (1980). Developmental changes in the effects of landmarks on infant spatial behavior. Developmental Psychology, 16, 312-318.

Aymed, A., \& Ruffman, T. (1998). Why do infants make A not B errors in a search task, yet show memory for location of hidden objects in a nonsearch task? Developmental Psychology, 34, 441-453.

Bai, D. L., \& Bertenthal, B. I. (1992). Locomotor status and the development of spatial search skills. Child Development, 63, 215-226.

Baillargeon, R. (1987). Object permanence in $3 \frac{1}{2}$ and $4 \frac{1}{2}$ month-oldinfants. Developmental Psychology, 23, 655-664.

Baillargeon, R., DeVos, J., \& Graber, M. (1989). Location memory in 8-month-old infants in a non-search A not B task: Further evidence. Cognitive Development, 4, 345-367.

Baillargeon, R., \& Graber, M. (1988). Evidence of location memory in 8 month old infants in a non-search AB task. Developmental Psychology, 24, 502-511.

Benson, J. B., \& Uzgiris, I. C. (1985). Effect of self-initiated locomotion on infant search activity. Developmental Psychology, 2I, 923-931.

Bertenthal, B. I., Campos, J. J., \& Barrett, K. C. (1984). Self-produced locomotion: An organizer of emotional, cognitive, and social development in infancy. In R. N. Emde \& R. J. Harmon (Eds.), Continuities and discontinuities in development (pp. 175-210). New York: Plenum.

Bjork, E. L., \& Cummings, E. M. (1984). Infant search errors: Stage of concept development or stage of memory development? Memory \& Cognition, 12, 1-19.

Bower, T. G. R., \& Paterson, J. G. (1971). Stages in the development of the object concept. Cognition, 1, 47-55.

Bremner, J. G. (1978a). Egocentric versus allocentric spatial coding in 9-month-old infants: Factors influencing the choice of code. Developmental Psychology, 14, 346-355.

Bremner, J. G. (1978b). Spatial errors made by infants: Inadequate spatial cues or evidence of egocentrism? British Journal of Psychology, 69, 77-84.

Bremner, J. G., \& Bryant, P. E. (1977). Place versus response as the basis of spatial errors made by young infants. Journal of Experimental Child Psychology, 23, 162-171.

Bremner, J. G., Knowles, L., \& Andreasen, G. (1994). Processes underlying young children's spatial orientation during movement. Journal of Experimental Child Psychology, 57, 355-376.

Bushnell, E. W., McKenzie, B. E., Lawrence, D. A., \& Connell, S. (1995). The spatial coding strategies of one-year-old infants in a locomotor search task. Child Development, 66, 937-958.

Butterworth, G. (1976). Object identity in infancy: The interaction of spatial location codes in determining search errors. Child Development, 46, 866-870.

Butterworth, G. (1977). Object disappearance and error in Piaget's Stage IV task. Journal of Experimental Child Psychology, 23, 391-401.

Butterworth, G., Jarrett, N., \& Hicks, L. (1982). Spatiotemporal identity in infancy: Perceptual competence or conceptual deficit? Developmental Psychology, 18, 435-449.

Cornell, E. H. (1981). The effects of cue distinctiveness on infants' manual search. Joumal of Experimental Child Psychology, 32, 330-342.

Cornell, E. H., \& Heth, C. D. (1979). Response versus place learning by human infants. Journal of Experimental Psychology: Human Learning and Memory, 5, 188-196.

Corrigan, R., \& Fischer, K. (1985). Controlling sources of variation: A skill theory approach. In H. M. Wellman (Ed.), Children's searching: The development of spatial skill and spatial representation (pp. 287-318). Hillsdale, NJ: Erlbaum.

Cutting, J. E. (1986). Perception with an eye for motion. Cambridge, MA: MIT Press.

Evans, W. F., \& Gratch, G. (1972). The stage IV error in Piaget's theory of object concept development: Difficulties in object conceptualization or spatial location? Child Development, 43, 682-688.

Flavell, J. H. (1963). The developmental psychology of Jean Piaget. New York: Van Nostrand.

Gibson, J. J. (1966). The senses considered as perceptual systems. Boston: Houghton Mifflin.

Goldfield, E. C., \& Dickerson, D. J. (1981). Keeping track of locations during movement of 8- to 10-month-old infants. Joumal of Experimental Child Psychology, 32, 48-64.

Gratch, G., Appel, K. J., Evans, W. F., LeCompte, G. K., \& Wright, N. A. (1974). Piaget's stage IV object concept error: Evidence of forgetting or object conception. Child Development, 45, 71-77.

Gratch, G., \& Landers, W. F. (1971). Stage IV of Piaget's theory of infants' object concepts: A longitudinal study. Child Development, 42, 359-372.

Harris, P. L. (1977). The child's representation of space. In G. Butterworth (Ed.), The child's representation of the world (pp. 83-93). New York: Plenum.

Hermer, L., \& Spelke, E. (1994). A geometric process for spatial reorientation in young children. Nature, 370, 57-59.

Hofstadter, M., \& Reznick, J. S. (1996). Response modality affects human infant delayed-response performance. Child Development, 67, 646-658.

Horobin, K., \& Acredolo, L. P. (1986). The role of attentiveness, mobility history, and separation of hiding sites on Stage IV search behavior. Joumal of Experimental Child Psychology, 41, 114-127.

Landau, B., \& Spelke, E. (1985). Spatial knowledge and its manifestations. In H. M. Wellman (Ed.), Children's searching: The development of search skill and spatial representation (pp. 27-52). Hillsdale, NJ: Erlbaum.

Landau, B., \& Spelke, E. (1988). Geometric complexity and object search in infancy. Developmental Psychology, 4, 512-521.

Laskey, R. E., Romano, N., \& Wenters, J. (1980). Spatial localization in children after changes in position. Journal of Experimental Child Psychology, 29, 225-248. 
Lee, D. N. (1993). Body-environment coupling. In U. Neisser (Ed.), The perceived self: Ecological and interpersonal sources of self-knowledge (pp. 43-67). Cambridge, England: Cambridge University Press.

Lee, D. N., \& Aronson, E. (1974). Visual proprioceptive control of standing in human infants. Perception \& Psychophysics, 15, 529-532.

Lee, D. N., \& Lishman, J. R. (1977). Visual control of locomotion. Scandinavian Journal of Psychology, 18, 224-230.

Lishman, J. R., \& Lee, D. N. (1973). The autonomy of visual kinaesthesis. Perception, 2, 287-294.

Lucas, T. C. \& Uzgiris, I. C. (1977). Spatial factors in the development of the object concept. Developmental Psychology, 13, 492-500.

Marcovitch, S., \& Zelazo, P. D. (1999). The A-not-B error: Results from a logistic meta-analysis. Child Development, 70, 1297-1313.

McKenzie, B. E. (1990). Early cognitive development: Notions of objects, space, and causality in infancy. In C.-A. Hauert (Ed.), Developmental psychology: Cognitive, perceptuo-motor and neuropsychological perspectives (pp. 43-60). Amsterdam: North-Holland.

Millar, S. (1993). Understanding and representing space: Theory and evidence from studies with blind and sighted children. Oxford, England: Clarendon Press.

Newcombe, N., Huttenlocher, J., Drummey, A. B., \& Wiley, J. G. (1998). The development of spatial location coding: Place learning and dead reckoning in the second and third years. Cognitive Development, 13, $185-200$.

Piaget, J. (1954). The construction of reality in the child. New York: Basic Books.

Pick, H. L., \& Lockman, J. J. (1981). From frames of reference to spatial representation. In L. S. Liben, A. H. Patterson, \& N. Newcombe (Eds.), Spatial representation and behavior throughout the life-span (pp. 3961). New York: Academic Press.

Presson, C. C., \& Ihrig, L. M. (1982). Using mother as a spatial landmark: Evidence against egocentric coding in infancy. Developmental Psychology, 18, 699-703.

Presson, C. C., \& Somerville, S. C. (1985). Beyond egocentrism: A new look at the beginnings of spatial representation. In H. M. Wellman (Ed.), Children's searching: The development of spatial skill and spatial representation (pp. 1-26). Hillsdale, NJ: Erlbaum.

Rider, E. A., \& Rieser, J. J. (1988). Pointing at objects in other rooms: Young children's sensitivity to perspective after walking with and without vision. Child Development, 59, 480-494.

Rieser, J. J. (1979). Spatial orientation of six-month-old infants. Child Development, 50, $1078-1087$.
Rieser, J. J. (1983). The generation and early development of spatial inferences. In H. L. Pick \& L. P. Acredolo (Eds.), Spatial orientation in natural and experimental settings (pp. 39-71). New York: Plenum.

Rieser, J. J. (1989). Access to knowledge of spatial structure from novel points of observation. Journal of Experimental Psychology: Learning, Memory, and Cognition, 15, 1157-1165.

Rieser, J. J., Garing, A. E., \& Young, M. F. (1994). Imagery, action, and young children's spatial orientation: It's not being there that counts, it's what one has in mind. Child Development, 65, 1262-1278.

Rieser, J. J., Guth, D. A., \& Hill, E. (1986). Sensitivity to perspective structure while walking without vision. Perception, 15, 173-188.

Rieser, J. J., \& Heiman, M. L. (1982). Spatial self-reference systems and shortest-route behavior in toddlers. Child Development, 53, 524-533.

Rieser, J. J., \& Rider, E. A. (1991). Young children's spatial orientation with respect to multiple targets when walking without vision. Developmental Psychology, 27, 97-107.

Smith, L. B., Thelen, E., Titzer, R., \& McLin, D. (1999). Knowing in the context of acting: The task dynamics of the A-not-B error. Psychological Review, 106, 235-260.

Sophian, C., \& Wellman, H. M. (1983). Selective information use and perseveration in the search behavior of infants and young children. Journal of Experimental Child Psychology, 35, 369-390.

Tyler, D., \& McKenzie, B. E. (1990). Spatial updating and training effects in the first year of human infancy. Journal of Experimental Child Psychology, 40, 445-461.

Warren, W. H., \& Hannon, D. J. (1988). Direction of self-motion is perceived from optical flow. Nature, 336, 162-163.

Warren, W. H., Mestre, D. R., Blackwell, A. W., \& Morris, M. W. (1991). Perception of circular heading from optical flow. Journal of Experimental Psychology: Human Perception and Performance, 17, 28-43.

Warren, W. H., Morris, M. W., \& Kalish, M. (1988). Perception of translational heading from optical flow. Journal of Experimental Psychology: Human Perception and Performance, 14, 644-660.

Wellman, H. M., Cross, D., \& Bartsch, K. (1986). Infant search and object permanence: A meta-analysis of the A-not-B error. Monographs of the Society for Research in Child Development, $5 /(3$, Serial No. 214).

Received July 28, 1998

Revision received February 4, 2000 Accepted February 9, 2000 


\section{Movement Type}

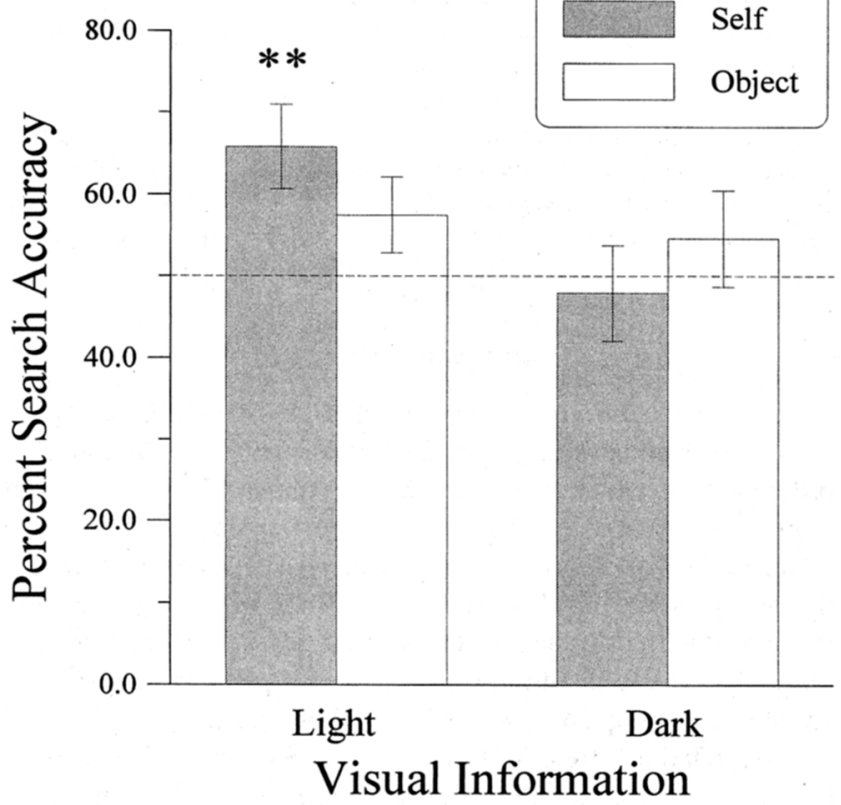

